Historic, Archive Document

Do not assume content reflects current scientific knowledge, policies, or practices. 



\section{WELLINGTON, KANSAS}

\section{PRICE LIST FOR SPRING 1922}

By freight or express, purchaser paying charges. If you desire to have Roses, ornamental shrubs, or other plants go forward by mail, add $5 \mathrm{c}$ postage to the prices in this list for each plant.

Kindly give full directions as to shipping, whether by freight, express, or mail. Our perfect pack. ing of stock admits of a long journey of stock without injury to same so that you neel not hesitate to order all heavy trees in large amounts shipped by freight. The freight rates are exceptionally low on trees. On all small bales up to about 50 pounds the express rates are often cheaper, and so much quicker that we especially recommend shipping by express. Trees take low second class express rates or one-half merchandise rates.

We guarantee all stock to be first-class, up to the size and grade specified in this list. Any claim should reach us within five days after receipt of stock, when same will receive prompt consideration.

Our stock is inspected by the State Inspector every year and certificates of inspection will be furnished with every shipment, showing the stock to be entirely free from San Jose Scale, or other injurious insects or diseases. In filling orders from this list we reserve the right in case we are out of the variety to substitute another of equal or better merit, labeling with correct name, unless the party says we shall not substitute in which case we will fill the order as far as we can and refund the balance.

TERMSi-Our terms are net cash with order. Five at 10 rates, and 50 at 100 rates.

APPLES AND CRABS

$$
\text { Each } \quad 10 \quad 100
$$

$\mathrm{X}, 2$ to $3 \mathrm{ft}$.............\$ $.50 \$ 4.80 \$ 46.00$ XX. 3 to $4 \mathrm{ft} \ldots \ldots . . . . . . .80 \quad 7.50 \quad 70.00$ $\mathrm{XXX}, 4$ to $6 \mathrm{ft} . \ldots . . . .1 .00 \quad 9.00 \quad 85.00$ Summer:-Red June, Ey. Harvest, Yellow Transparent, Maiden Blush, Wealthy.

Fall and Winter:- Jonathan, Grimes Golden, Delicious, Stayman Winesap, Rome Beauty, Tolman Sweet, Huntsman, Ben Davis, Gano (Black Ben), Winter Banana, York Imperial, Winesap, Mammoth Black Twig, Geniton, Hyslop Crab, Whitnoy Crab.

\section{PEARS (Standard and Dwarf)}

\begin{tabular}{lrrr} 
& Each & \multicolumn{1}{c}{10} & \multicolumn{1}{c}{100} \\
$\mathrm{X}, 2-3 \mathrm{ft} \ldots \ldots \ldots \ldots \ldots . \$ .75$ & $\$ 7.00$ & $\$ 65.00$ \\
$\mathrm{XX}, 3-4 \mathrm{ft} \ldots \ldots \ldots \ldots \ldots \ldots .1 .00$ & 1.50 & 90.00 \\
$\mathrm{XXX}, 4-6 \mathrm{ft} \ldots \ldots \ldots \ldots \ldots .25$ & 12.20 & 120.00
\end{tabular}

Varieties:-Bartlett, Seckel, 'Duchess, Garber, Keiffer.

\section{CHERRIES}

$$
\text { Each } \quad 10 \quad 100
$$

X. $2-3$ ft...............\$.80 $\$ 7.50 \quad \$ 70.00$ XY. 3-4 ft.................1.00 $9.50 \quad 90.00$ XXY, 4-6 ft..............1.50 14.00 130.00 Varieties:-Early Richmond, Montmorency, English Morillo, May Duke, Dyehouse, Wragg and Compass.

\section{APRICOTS}

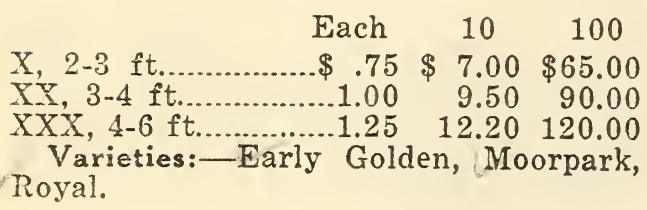

\begin{tabular}{|c|c|c|c|}
\hline & Each & 10 & 100 \\
\hline $\mathrm{X}, 2-3$ ft.... & $\ldots \$ .75 \$$ & 7.00 & $\$ 65.00$ \\
\hline $\mathrm{XXX}, 4-6 \mathrm{ft}$ & $\ldots .1 .25$ & 12.20 & 120.00 \\
\hline
\end{tabular}

\section{PLUMS}

Varieties:-Abundance, Burbank, Red June, Imperial Gage, Wild Goose, Wickson, Shropshire Damson.

\section{PEACHES}

$$
\text { Each } 10
$$

X. 2-3 ft............... \$ $.50 \$ 4.80 \$ 46.00$

$\mathrm{XX}, 3-4 \mathrm{ft} . \ldots . \ldots \ldots \ldots . . . . . . .80 \quad 7.50 \quad 70.00$

XXX, 4-6 ft...............1.00 $9.00 \quad 85.00$

Varieties:- Greensboro, Carmen, Belle of Georgia, Champion, Early Crawford, Capt. Ede Fitzorerald, Elberta, Stump, Chinese Cling, Henrietta, Heath Cling, Wonderful, Mayflower, Japan Blood Dwarf, Late Crawford.

\section{GOOSEBERRIES}

Each 40 c; 10 for $\$ 3.75 ; 100$ for $\$ 35.00$ Downing and-Houghton, the two best varieties.

\section{CURRANTS}

White Dutch and Fay's Prolific, price same as Gooseberries.

\section{GRAPES}

Each 40 c; 10 for $\$ 3.80 ; 100$ for $\$ 35.00$ Yarieties:-Concord, - Moore's Early Brighton, Diamond, Worden, Niagara.

\section{BLACKBERRIES}

Each 12 c; 10 for $\$ 1.00 ; 100$ for $\$ 8.00$ 1,000 for $\$ 75.00$

Varieties:- Early Harvest, Snyder, Mersereau.

Lucretia Dewberies, same price as Blackberries. 
Each $12 \mathrm{c} ; 10$ for $\$ 1.00 ; 100$ for $\$ 8.00$ 1,000 for $\$ 75.00$.

Varieties:-Cuthbert, Kansas, Gregg, and'St. Regis.

\section{GARDEN ROOTS}

\begin{tabular}{|c|c|c|}
\hline Each & 10 & 100 \\
\hline Rhubarb ...... & $\$ 1.25$ & $\$ 10.00$ \\
\hline Asparagus ................ 06 . & .50 & 4.00 \\
\hline Horseradish ............. 10 & .75 & 6.50 \\
\hline
\end{tabular}

\section{STRAWBERRIES}

(By Parcel Post or Express Prepaid) 25
50
100
250
500

$\$ .70 \quad \$ 1.00 \quad \$ 1.75 \quad \$ 3.00 \quad \$ 5.00$

Varieties:- Senator Dunlap, Aroma, Gandy, 'Warfield, Klondyke.

Fall Bearing Strawberries

$25 \quad 50 \quad 100 \quad 250 \quad 500$

$\$ 1.25 \quad \$ 2.00 \quad \$ 3.50 \quad \$ 6.00 \quad \$ 10.00$ Progressive bears loads of Strawberries until killed by frost.

\section{ORNAMENTAL TREES}

\section{Each}

10

Catalpa Bungeii, 2 yr. heads..........................\$3.00 $\$ 25.00$

Catalpa Bungeii, 1 yr. heads..............................2.50 22.50

Mulberry, Tea's Weeping......4.00 35.00

Ash, American White,

$$
\text { 4-5 ft........................... .50 }
$$

Ash, American White,

$$
\text { 5-6 ft........................... .75 }
$$

Ash, American White,

$$
\text { 6-8 ft............................... } 1.00
$$

7.00

Bechtel's Flowering Crab,

$$
\text { 2-3 ft............................. } 1.00
$$

Maple, Silver or Soft.

$$
\text { 4-5 ft. }
$$

Maple, Silver or Soft.

$$
\text { 5-6 ft.......................... .75 }
$$

Maple, Silver or Soft. 6-8 $\mathrm{ft}$.

Maple, Silver or Soft.

$$
\text { 8-10 ft............................ } 1.50
$$

Maple, Silver or Soft.

$$
\text { 10-12 ft........................2. } 2.00
$$

Poplar, Carolina, 4-6 ft...... .50

Poplar, Carolina, 6-8 ft......... .60

Poplar, Carolina, 8-10 ft...... .75

Poplar, Carolina, 10-12 ft.....1.25

Poplar, Lombardy, 4-6 ft....... .60

Poplar, Lombardy, 6-8 ft...... . .75

Mulberry, Russian, 4-6 ft.... .50

Mulberry, Russian, 6-8 ft.... .75

Elm; American, 4-6 ft......... .50

Elm, American, 6-8 ft.........1.00

Elm, American, 8-10 ft........1.50

Elm, American, 10-12 ft.......2.00

Catalpa, Speciso, 4-6 ft....... .50

Catalpa, Speciosa, 6-8 ft. ....1.00

Catalpa, Speciosa, 8-10 ft.....1.50

Catalpa, Speciosa, 10-12 ft...2.00

Hackberry, 6-8 ft.................1.00

Hackberry, 8-10 ft.
9.00

9.00

4.00

6.50

9.00

12.50

15.00

4.00

5.00

6.00

10.00

5.00

7.00

4.00

6.00

4.00

9.00

12.00

15.00

4.00

9.00

12.50

15.00

9.00

12.50
California Privett, 12-24 in

100

Amoor River North Privett, $12-24$ in

$\$ 15.00$

20.00

Spirea Van Houtteii, 12-24 in.......50.00

Japanese Barberry, 12-24 in.........45.00

Bush Honeysuckle, 12-24 in..........50.00

Osage Hedge, 12-24 in.

\section{EVERGREENS}

Chinese Arbor Vitae,

$$
\text { Each } 10 \quad 100
$$

12 in.................. \$ .60 \$ $5.50 \$ 50.00$

Chinese Arbor Vitae,

18 in...................... $80 \quad 7.50 \quad 70.00$

Chinese Arbor Vitae,

$2 \mathrm{ft} \ldots \ldots . . . . . . . . . . . . .1 .00 \quad 9.00 \quad 85.00$

Austrian Pine 12-in. 1.00

Austrian Pine 18-in. 1,25

Austrian Pine, 2 ft...1.50

Scotch Pine, 12-in...1.00

Scotch Pine, 18-in...1.25

Scotch Pine, 2 ft.....1.50

Red Cedar, 10-in..... .60

Red Cedar, 12-in..... .80

9.00

11.50

14.00

9.00

11.50

14.00

5.50

7.50

50.00

Red Cedar, 18-in.....1.20 11.00

Red Cedar, 2 ft..........1.60 15.00

70.00

\section{SHRUBS}

Spirea Van Houtteii........... \$ .75

Spirea Anthony Waterer..... . .75

Barberry, Japanese.............. .75

Japanese Quince.................... .75

Mock Orange, or Syringa.... .75

Snowball .............................. .75

Bush Honeysuckle................. .75

Lilac .................................... .50

Althea ................................... .75

Rosa Rugosa............................. .75

Privett, Armoor North......... . .25

Privett, Regal's...................... .50

\section{ORNAMENTAL VINES}

American Ivy.

Each

Clematis (Purple or White) 1.00

10

Honeysuckle (Red Trumpet) .75

Honeysuckle (Hall's Japan) .75

Wisteria (Chinensis)

.75

$\$ 4.50$

9.00

7.00

7.00

7.00

\section{HARDY ROSES}

$\begin{array}{cc}\text { Each } & 10 \\ \text { Hybrid Perpetual Roses...... } \$ 1.00 & \$ 9.00 \\ \text { Varieties:-Coquet des Alps, } & \text { Frau }\end{array}$ Karl Druschki, General Jackqueminot, Paul Neyron, Ulrich Brunner, Eugene Furst, Madam Plentier, Marshal P. Wilder.

Hardy Climbing Roses........\$.80 $\$ 7.50$ Varieties:-Crimson Rambler, Pink Dorothy Perkins, Excelsa or Red Dorothy Perkins, White Dorothy Perkins, Baltimore Belle, Queen of the Prairies, Yellow Rambler. 


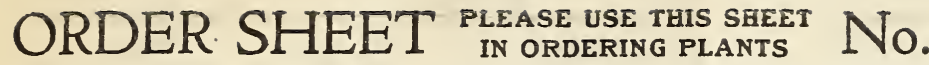

HOW TO ORDER Fill out this Order Sheet with the items you want to buy, enclosing Postal Money Order, Bank Draft or Check, and mail all to us in enclosed envelope. By this method you save half the money you would have to pay agents for trees. We have filled orders in this way for years, and are known all through the country. You can learn of our responsibility through any commercial agency, bank or banker, or of the postmaster at Wellington, Kans. We pack and ship all Winter to Southern States. Our Regular Spring Shipping Season opens about March 15. State positively how we are to ship. Whether by Freight, Express or Mail. If by Freight or Express, give name of R. R. station and your postoffice. If you desire plants shipped by mail, add 5 cents per plant to list price.

\section{WELLINGTON NURSERIES}

WEI.I.INGTON, KANSAS

Name.

Post Office

County.

State.

Be sure to give name of Express Office if desired by Express, or Freight Station if desired by Freight. Also name your Post Office Address.

Express Office.

Freight Station.

Express Co.

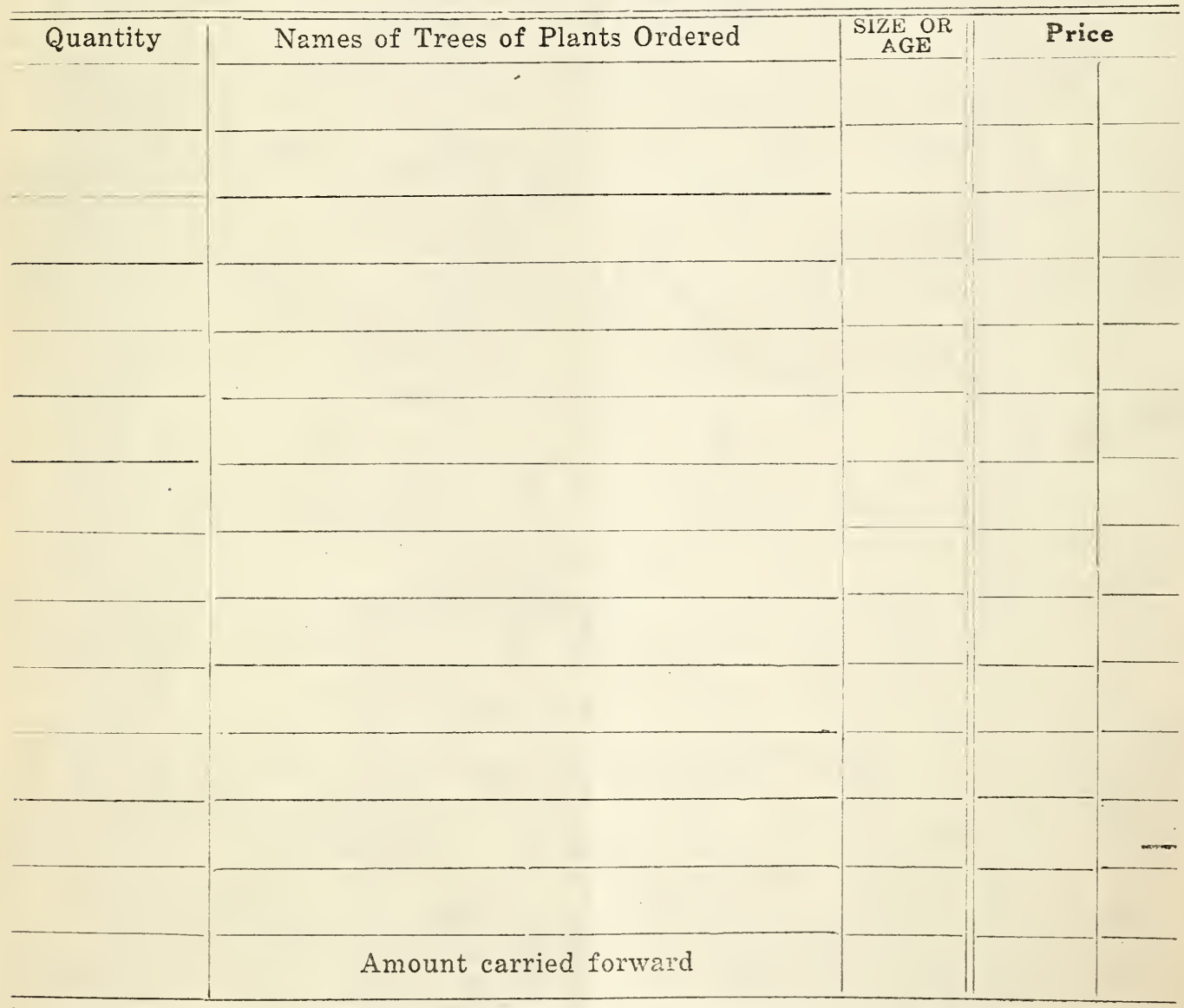

OUR GUARANTEE:-While we exercise the greatest care to have our stock genuine and reliable, we agree to replace on proper proof, all that may prove untrue to name as labeled, and it is mutually agreed that our replacing it or of fer to replace it shall operate as a liquiciation of all damages.

No Charge For Boxing at Catalog Prices 


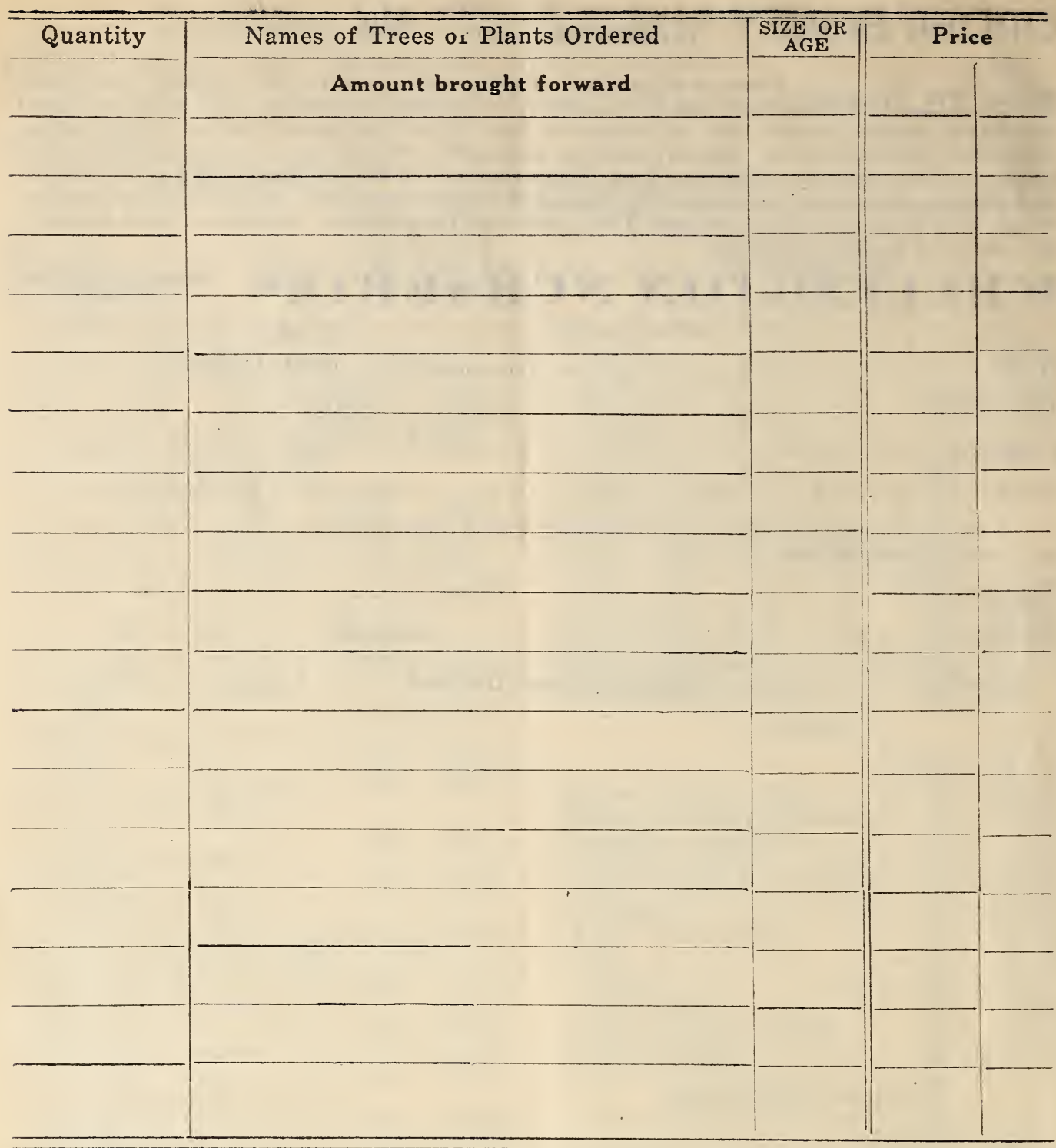

Please write below the names and addresses of a few of your friends who are interested in, and buyers of TREES, PLANTS, ETC. 